in these mice. We studied mutant actin by an in-vitro motility assay. Thin filaments were reconstituted with purified mouse $\mathrm{f}$-actin and human heart tropomyosin and troponin. The E99K thin filaments had higher $\mathrm{Ca}^{2+}$ sensitivity than non-transgenic thin filaments. E99K actin thin filaments did not respond to troponin dephosphorylation. The ACTC E99K mouse reproduces many features of HCM, as observed in patients. The basic effect of the ACTC E99K mutation is increased $\mathrm{Ca}^{2+}$ sensitivity together with a blunted response to troponin I phosphorylation. The increased myofibrilar $\mathrm{Ca}^{2+}$ sensitivity may be sufficient to provoke arrhythmia and account for the high mortality at early ages. Hypertrophy may be a chronic response to $\mathrm{Ca}^{2+}$ overloading or due to energy depletion.

\section{HYPOXIC PRECONDITIONING OF CARDIOSPHERE-DERIVED CELLS TO INCREASE RETENTION IN THE INFARCTED HEART}

doi:10.1136/hrt.2009.191064j

S C Tan, L Le Page, L C Heather, J J Tan, D J Stuckey, C A Carr, K Clarke. Department of Physiology, Anatomy and Genetics, University of Oxford, Oxford OX1 3PT, UK

Myocardial infarction results in the formation of a hypoxic scar region. Resident stem cells have been discovered in the adult heart that may be expanded in vitro via the formation of cardiospheres. Administration of these cardiosphere-derived cells (CDC) to the infarcted heart has been shown to improve cardiac function; however, levels of stem cell retention are low. Preconditioning of CDC to a hypoxic environment may increase cell retention, promote proliferation within the scar and further improve cardiac function. CDC were cultured under $2 \%$ oxygen for 1 week. Proliferation rates were calculated and hypoxic inducible factor (HIF1 $\alpha$ ) protein expression and oxygen consumption were measured in intact cells over 1 week. CDC culture under hypoxia for $24 \mathrm{~h}$ increased HIF1 $\alpha$ by $214 \%$ compared with control cells cultured under normoxia. After 1 week in hypoxia, however, there was no difference in HIF1 $\alpha$ levels compared with controls. CDC proliferation was increased fivefold under hypoxia. CDC cultured under hypoxia had decreased oxygen consumption compared with control cells cultured under normoxia, with oxygen consumption decreased by $22 \%$ with both ADP and FCCP after $24 \mathrm{~h}$. After 1 week of hypoxia, oxygen consumption was decreased by $92 \%$ with $\mathrm{ADP}$ and $94 \%$ with FCCP. Culture under hypoxia generated sufficient $\mathrm{CDC}$ for therapy more rapidly than under normoxia. The resulting CDC had reduced oxygen consumption and thus may be better adapted to survive within the hypoxic scar.

\section{TRANSCRIPTIONAL REGULATION OF P40PHOX AND P47PHOX EXPRESSION VIA HBP1 IN ENDOTHELIAL CELLS}

doi:10.1136/hrt.2009.191064k

L Teng, J-M Li. Cardiovascular Research Theme, Faculty of Health and Medical Sciences, University of Surrey, UK

Transcription factor HMG-box protein 1 (HBP1) is a member of the HMG-box family of transcription factors and has been found to play an important role in the transcriptional repression of the p47phox gene. The promoter region of p40phox also has a HBP1 binding site, which makes p40phox a possible candidate for HBP1. In this study, we examined the role of HBP1 in the regulation of $\mathrm{p} 47$ phox and p40phox in endothelial cells. Knockdown of p47phox in a mouse lymphoid endothelial cell line (SVEC4-10) resulted an $\sim 50 \%$ increase of HBP1 protein expression, and this was accompanied with a significant increase in $\mathrm{p} 40$ phox protein expression as detected by Western blot. The levels of HBP1 expression were significantly higher
( $\sim 2.3$-fold) in coronary microvascular endothelial cells isolated from p47phox knockout mice compared with cells isolated from wild-type mice. The role of HBP1 in the transcriptional regulation of p40phox and p47phox expression was further examined by transient in-vitro knockdown of HBP1 using shRNA in human microvascular endothelial cell (HMEC1). Knockdown of HBP1, as shown by Western blot, resulted in a significant increase in $\mathrm{p} 47$ phox expression and this was accompanied with a significant reduction in p40phox expression. In conclusion, HBP1 plays dual roles in the regulation of $\mathrm{NADPH}$ oxidase: it represses $\mathrm{p} 47 \mathrm{phox}$ expression and in the mean time promotes p40phox expression. HBP1 may represent an important transcriptional mechanism involved in the regulation of endothelial reactive oxygen species production by NADPH oxidase.

\section{ENDOTHELIAL NOX4 NADPH OXIDASE ENHANCES VASODILATATION VIA HYDROGEN PEROXIDE-INDUCED HYPERPOLARISATION AND REDUCES BLOOD PRESSURE}

doi:10.1136/hrt.2009.191064

M Wang, R Ray, C Murdoch, M Zhang, S J Walker, A C Brewer, A M Shah. King's College London BHF Centre of Excellence, The James Black Centre, London, UK

Introduction NADPH oxidases (Noxs) are reactive oxygen speciesgenerating enzymes implicated in cardiovascular disease. Nox4 is the most abundantly expressed isoform in endothelial cells but its function remains unknown. We investigated the role of endothelial Nox4 on vascular function and blood pressure (BP) in vivo.

Methods and Results We generated transgenic mice with endothelium-specific overexpression of Nox4 (Nox4TG) and studied the effects on endothelial function (aortic rings ex vivo) and blood pressure (telemetry). Nox4 protein levels were twofold higher in Nox4TG aorta compared with wild-type (wt) littermates, with no changes in the expression of other Nox isoforms. Nox4TG had enhanced relaxation to acetylcholine (ACh) compared with wt mice

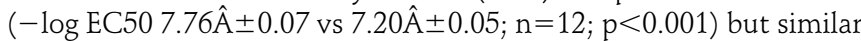
relaxation to sodium nitroprusside. The ACh response in Nox4TG and wt mice was dentical in the presence of catalase $(1500 \mathrm{U} / \mathrm{ml})$ or with high extracellular potassium $(30 \mathrm{mM})$ pre-contraction, but remained greater in Nox4TG in the presence of inhibitors of nitric oxide synthesis (L-NMMA, 100ÂAM), soluble guanylate cyclase (ODQ, $5 \hat{A} \mu M)$ or protein kinase $G(K T 5823,2 \hat{A} \mu M)$. Ox4TG also had significantly lower BP than wt mice (mean BP $102.5 \hat{\mathrm{A}} \pm 1.8$ vs $109.5 \hat{\mathrm{A}} \pm 2.0 \mathrm{~mm} \mathrm{Hg} ; \mathrm{n}=10 ; \mathrm{p}=0.05$ ), which was abolished after chronic treatment with $\mathrm{N}$-acetylcysteine or an $\mathrm{OD} /$ catalase imetic, EUK-8. Plasma nitrite/nitrate levels and aortic levels of phosphorylated VASP were identical and acute intravenous treatment with $\mathrm{L}$ NMMA $(10 \mathrm{mg} / \mathrm{kg})$ increased BP to a similar extent in Nox4TG and wt mice. The hypertensive response to chronic 14-day angiotensin II infusion (1.1 $\mathrm{mg} / \mathrm{kg}$ per day) was lower in ox4TG compared with wt mice (mean BP $116.7 \hat{\mathrm{A}} \pm 4.7$ vs $129.4 \hat{\mathrm{A}} \pm 3.5 \mathrm{~mm} \mathrm{Hg} ; \mathrm{n}=10 ; \mathrm{p}<0.05$ ). Conclusions Nox4TG had significantly enhanced ACh-induced vasodilatation compared with wt mice as a result of hydrogen peroxideinduced hyperpolarisation. Nox4TG also had a lower BP, which was not attributable to altered nitric oxide bioactivity but was normalised by chronic antioxidant treatment. These results suggest that endothelial Nox4 has potentially beneficial effects on vascular tone and BP.

\section{IS DEPRESSED MYOCYTE CONTRACTILITY AN EARLY EVENT IN THE NATURAL HISTORY OF HEART FAILURE?}

doi:10.1136/hrt.2009.191064m

W Idigo, Y H Zhang, C Lygate, B Casadei. Department of Cardiovascular Medicine, University of Oxford, John Radcliffe Hospital, Oxford, UK

It is generally accepted that abnormal intracellular $\mathrm{Ca}^{2+}$ handling accounts for the depressed left ventricular (LV) systolic and diastolic 
function in failing hearts. These findings have sparked a number of experimental and clinical studies aimed at restoring intracellular $\mathrm{Ca}^{2+}$ homoeostasis using viral gene transfer of $\mathrm{Ca}^{2+}$ transporter to the human myocardium; however, significant abnormalities in $\mathrm{Ca}^{2+}$ handling characterise late-stage heart failure and may not be the initiating factor. To test this hypothesis, we compared LV myocytes from C57BL6 mice 12 weeks after coronary ligation (causing an infarct $>35 \%$ by $3 \mathrm{D}$ echocardiography) or sham surgery. LV ejection fraction was significantly reduced in infarcted mice versus shams ( $23 \%$ vs $55 \%$, $\mathrm{p}<0.0001, \mathrm{n}=9$ in each group) and the heart weight or lung weight to body weight ratios were significantly increased. LV myocytes isolated from infarcted hearts were significantly larger than those from sham operated hearts $(p=0.001)$. The distribution of cell shortening and cell size measured in 'unselected' LV myocytes (ie, from all the live cells in a given field) was much wider in infarcted mice than in sham-operated controls. However, basal cell shortening $(3 \mathrm{~Hz}$, field stimulation at $35^{\circ} \mathrm{C}$ ) was enhanced in myocytes from infarcted hearts $(p=0.001)$, as was the amplitude of the $\left[\mathrm{Ca}^{2+}\right]_{i}$ transient $(p<0.0001)$ and the SR Ca content $(10 \mathrm{mM}$ caffeine spritz, $\mathrm{p}=0.04)$. The rate of decay of $\left[\mathrm{Ca}^{2+}\right]_{\mathrm{i}}$ was faster both in field-stimulated $(p<0.0001)$ and caffeine-induced $\left[\mathrm{Ca}^{2+}\right]_{\mathrm{i}}$ transients, suggesting upregulation of the $\mathrm{Na}^{+} / \mathrm{Ca}^{2+}$ exchanger activity. The myocyte inotropic response to increasing doses of isoproterenol or stimulation frequency $(0.5-3 \mathrm{~Hz})$ was similar, despite significant LV dysfunction and reduced $\beta$-adrenergic reserve in vivo. In summary, impaired LV performance post-myocardial infarction can occur in the absence of obvious $\mathrm{Ca}^{2+}$ and contraction abnormalities at the single myocyte level. These findings suggest that relatively minor changes in myocardial architecture, in terms of the distribution of cell properties, may lead to significant LV dysfunction and to the syndrome of heart failure well before individual myocytes show a significant deficit in contractility.

\section{PLASMA LEVELS OF THE NOVEL VASODILATORY GAS HYDROGEN SULPHIDE ARE ASSOCIATED WITH ADIPOSITY}

doi:10.1136/hrt.2009.191064n

M Whiteman, K M Gooding, J L Whatmore, K Skinner, C Ball, J E Tooke, A C Shore. Peninsula Medical School, St Luke's Ts Campus, Magdalen Road, Exeter, Devon, UK; Peninsula National Institute of Health Research Clinical Research Facility, Exeter, UK

Background Vascular synthesis of the novel endogenous vasodilatory and redox active gas hydrogen sulfide $\left(\mathrm{H}_{2} \mathrm{~S}\right)$ is perturbed in animal models of hypertension and diabetes. ${ }^{1}$ However, the role of H2S in the human vasculature in health or disease is unknown. We have investigated whether plasma H2S levels correlated to physical, clinical and biochemical indices of obesity and diabetes in men.

Methods Plasma was obtained from male patients with type 2 diabetes mellitus (T2DM; $n=11$ ), overweight (OW; $n=16)$ and lean $(\mathrm{n}=11)$ healthy male volunteers, and $\mathrm{H}_{2} \mathrm{~S}$ levels were determined by zinc-trap spectrophotometry. Anthropometric measurements (body mass index, waist-hip ratio), lipid profile, systemic blood pressure and indices of diabetes (fasting glucose, insulin sensitivity, glycated haemoglobin) were determined.

Results Mean plasma $\mathrm{H}_{2} \mathrm{~S}$ levels were significantly lower in T2DM patients (mean \pm SD $12.73 \pm 8.67 \mu \mathrm{M}$ ) compared with lean
$(41.1 \pm 17.9 \mu \mathrm{M}, \mathrm{p}=0.001 \mathrm{Mann}-$ Whitney $\mathrm{U}$ test) and OW (OW $22.93 \pm 7.96 \mu \mathrm{M}, \mathrm{p}=0.008)$ volunteers. Mean plasma $\mathrm{H}_{2} \mathrm{~S}$ levels in OW volunteers were significantly lower than in lean controls $(p=0.003)$. Waist-hip ratio was a significant independent predictor of plasma $\mathrm{H}_{2} \mathrm{~S}(\mathrm{R} 2=0.431, \mathrm{p}<0.001$; standardised $\beta-0.657, \mathrm{p}<0.001$ linear regression) in the whole group. This relationship was independent of diabetes status, which contributed $4 \%$ to the model $(\mathrm{R} 2=0.472)$. Waist-hip ratio remained an independent predictor of plasma $\mathrm{H}_{2} \mathrm{~S}$ when adjusted for systolic blood pressure, insulin sensitivity, glycaemic control and lipid profile. These findings were reflected with both body mass index and waist circumference.

Conclusions The reduction in plasma $\mathrm{H}_{2} \mathrm{~S}$ in both $\mathrm{OW}$ and T2DM male individuals is primarily driven by adiposity.

\section{REFERENCE}

1. Whiteman M, Moore. J Cell Mot Med 2009;13:488-507.

\section{PROTECTIVE ROLE OF PEROXISOME PROLIFERATOR-ACTIVATED RECEPTOR Ia/ $/ \mathrm{a}^{\prime}$ IN CARDIAC DYSFUNCTION AND ORGAN INJURY/INFLAMMATION CAUSED BY ENDOTOXIN IN MICE}

doi:10.1136/hrt.2009.1910640

A Kapoor, Y Shintani, M Collino, L K Bailey, N S A Patel, B Sepodes, P Tripatara, S Castiglia, R Fantozzi, H Mota-Filipe, K Suzuki, J A Mitchell, C Thiemermann. Centre for Translational Medicine and Therapeutics, William Harvey Research Institute, UK

Peroxisome proliferator-activated receptor (PPAR)- $\mathrm{I}^{\mathrm{a} 2} / \mathrm{I}^{\mathrm{a}^{\prime}}$ is a transcription factor that belongs to the PPAR nuclear hormone receptor family. There is little information about the ligands of PPAR- $\mathrm{I}^{\mathrm{a}} / \mathrm{I}^{\mathrm{a}}$ and the effect of specific activation (eg, GW0742) of PPAR- $\mathrm{I}^{2} / \mathrm{I}^{\mathrm{a}^{\prime}}$ in animal models of shock. Here we used PPAR-I $\mathrm{I}^{2} / \mathrm{I}^{\mathrm{a}^{\prime}}$ knockout (ko) mice to investigate the role of this transcription factor in a lipopolysaccharide-induced model of cardiac dysfunction and organ injury/ inflammation. When challenged with lipopolysaccharide $(9 \mathrm{mg} / \mathrm{kg}$ intraperitoneally) for $16 \mathrm{~h}$, ko mice exhibited a significant reduction in cardiac function when compared with wild-type (wt) mice, as revealed by echocardiography. When compared with wt mice, ko mice exhibited significantly increased serum levels of creatinine (renal dysfunction) and alanine aminotransferase (hepatocellular injury). In C57/BL6 mice, administration of the highly selective PPAR-I ${ }^{2} / \mathrm{I}^{\mathrm{a}^{\prime}}$ agonist GW0742 $(0.03 \mathrm{mg} / \mathrm{kg}$ intravenously $) 1 \mathrm{~h}$ after lipopolysaccharide challenge significantly attenuated the cardiac dysfunction caused by lipopolysaccharide-induced endotoxaemia, as revealed by echocardiography and the isolated-perfused Langendorff heart. Pre-treatment with a highly selective PPAR- $\mathrm{I}^{\mathrm{a}} / \mathrm{I}^{\mathrm{a}^{\prime}}$ antagonist GSK0660 $(0.1 \mathrm{mg} / \mathrm{kg}$ intravenously) 30 minutes before lipopolysaccharide reduced the cardioprotective effect of GW0742. When compared with mice challenged with lipopolysaccharide alone, GW0742 significantly attenuated creatinine serum levels and lung myeloperoxidase activity (lung inflammation). Our results suggest potential physiological ligands of PPAR- $\mathrm{I}^{2} / \mathrm{I}^{\mathrm{a}^{\prime}}$, which can afford a protective role in lipopolysaccharide-induced cardiac dysfunction and organ injury, as indicated by the loss of the receptor. Subsequently, activation of the receptor with a specific agonist, GW0742, inhibits the cardiac dysfunction and organ injury/inflammation caused by lipopolysaccharide-induced endotoxemia. 